\section{P141 Using Qualitative Research to Examine Factors Influencing Food Choices of Latinas While Enhancing Dietetic Student Experience}

Lisa Kessler, DrPH, MPH, RD, lakessler@csupomona.edu, California State Polytechnic University, Pomona, College of Agriculture, 3801 W. Temple Avenue, Pomona, CA 91768; C. Kerber, BS; S. Wallace, PhD, RD, MPH; B. BurnsWhitmore, DrPH, MPH, RD

Objective: It is increasingly important that registered dietitians working with the Latino population be prepared to provide culturally and linguistically appropriate nutrition care. The purpose of this study was to obtain information regarding usual dietary patterns of Latinas from Pomona, California while providing dietetics students with experience working with this community that they find valuable.

Study Design, Setting, Participants, and Intervention: Data were collected in three focus groups held in Spanish that discussed participant's eating patterns and dietary concerns. Undergraduate Spanish-speaking Estudiante de Dietético (ED, a US Department of Agriculture funded curriculum) students from the university's dietetics program were used to facilitate groups, translate and transcribe data. Focus groups occurred at two community centers in Pomona, CA that service surrounding low-income neighborhoods. Seventeen self-identified Latina women (aged $30-70$ ) that utilize services at their local community center participated in focus groups.

Outcome, Measures and Analysis: To analyze qualitative data, research team members reviewed transcribed data and identified themes using the constant comparative method. Students completed satisfaction surveys to assess usefulness of the activity.

Results: Focus group participants described traditional recipes and family preferences as main influences on foods prepared. Motivators for eating healthy include maintaining family health and preventing weight gain. Barriers include conflicting meal schedules and individual food preferences. Students reported greater knowledge of nutrition education for Latinos and increased confidence working with this population.

Conclusions and Implications: Integrating real-life learning experiences into the dietetics curriculum can provide mutual benefit to students and community members. Future educational initiatives should continue to incorporate work within the Latino community.

Funding: Agricultural Research Institute in California

\section{P142 Investigation of Food and Nutrition Management Program and its Needs for the Aged Using the Elderly Welfare Facility}

Youngmee Lee, PhD, leeym@gachon.ac.kr, Department of Food \& Nutrition, Gachon University, 1342 SeongnamDaro, Sujung-Gu, Seongnam-shi, Geonggi-Do, 462-701, South Africa; J. Kim, PhD, Paichai University; M. Lee, PhD, Yonsei University; O. Park, PhD, College of Yeoju; $H$. Yoo, MS, RD, Eulji University Hospital
Objective: This research was intended to investigate the food and nutrition management programs for the aged in the elderly welfare facility and its program needs of the aged.

Target Audience: The target audience was the elderly in community.

Theory, Prior Research, Rationale: The elderly welfare facility for enhancing the quality of lifehas been increased quantitatively in Korea. This trend implies that a social security-based approach to reducing social cost such as medical expenses of the aged could be an efficient method.

Description: We performed interviews with 399 elderlies for nutritional assessment,dietary/nutrition management and program needs by visiting public health centers and community halls for the aged.

Evaluation: It was represented that $80 \%$ of the total subjects were taking some prescribed drugs due to their retained diseases. By using Mini Nutritional Assessment (MNA) as a tool of nutritional screening for the aged, ratio of malnutrition risk group was $37.8 \%$. Problems of taking a meal were generally represented as high complaint for chewing difficulty (20.6\%) and indigestion $(13.8 \%)$, and major dietary problem was excessive intake of salt $(15.8 \%)$ When the aged select food, they consider 'taste $(45.9 \%)$ ' the most, but in case of welfare center, 'nutrition $(39.0 \%)$ ' was highly considered $(\mathrm{p}<0.001)$. For the food and nutrition management control system of the aged, it was represented that 'provision of nutritious food $(21.1 \%)$ ', 'nutrition \& health education $(19.3 \%)$ ' and 'provision of nutritionally balanced meal service $(17.5 \%)$ '.

Conclusions and Implications: It requires a management-based approach rather than treatment-based one for health of the elderly in community. Therefore, health promotion countermeasures for the aged through welfare facility is required.

Funding: Korea Health Promotion Foundation (2013)

\section{P143 Eating Habits, Practices Associated With Weight Loss and Nutrition Knowledge of Women Attending to Sport Centers}

Duygu Kozan, MS, duygu.kapikiran@hotmail.com, Orhanbey, Orhanbey, Bursa, 16600, Turkey; N. Aktas, PhD, Selcuk University

Objective: To determine eating habits, practices associated with weight loss and nutritional knowledge levels of women attending to sport center.

Study Design, Setting, Participants, and Intervention: A cross-sectional descriptive research design. The women $(n=200)$ who volunteered to participate in the study aged 18-64 years, attending to three sports center in Tokat, Turkey.

Outcome, Measures and Analysis: Women's demographic variables, eating habits, nutrition knowledge and practices associated with weight loss were assessed by a 